\title{
A UAV BASED 3-D POSITIONING FRAMEWORK FOR DETECTING LOCATIONS OF BURIED PERSONS IN COLLAPSED DISASTER AREA
}

\author{
H. Moon ${ }^{\text {a, } *}$, C. Kim ${ }^{\text {a }}$ W. Lee ${ }^{\text {a }}$ \\ ${ }^{a}$ Korea Institute of Civil Engineering and Building Technology, ICT Convergence and Integration Research Institute, \\ 283 Goyang-daero, Goyang-si, Gyeonggi-do, Republic of Korea, 10223 - (hsmoon, ckim, wslee)@kict.re.kr
}

Commission VIII, WG VIII/1

KEY WORDS: Drone, 3D Positioning Framework, Barometer, Wireless Sensor, Buried Person Detection

\begin{abstract}
:
Regarding spatial location positioning, indoor location positioning theories based on wireless communication techniques such as WiFi, beacon, UWB and Bluetooth has widely been developing across the world. These techniques are mainly focusing on spatial location detection of customers using fixed wireless APs and unique Tags in the indoor environment. Besides, since existing detection equipment and techniques using ultrasound or sound etc. to detect buried persons and identify survival status for them cause $2^{\text {nd }}$ damages on the collapsed debris for rescuers. In addition, it might take time to check the buried persons. However, the collapsed disaster sites should consider both outdoor and indoor environments because empty spaces under collapsed debris exists. In order to detect buried persons from the empty spaces, we should collect wireless signals with Wi-Fi from their mobile phone. Basically, the Wi-Fi signal measure 2-D location. However, since the buried persons have $\mathrm{Z}$ value with burial depth, we also should collect barometer sensor data from their mobile phones in order to measure $Z$ values according to weather conditions. Specially, for quick accessibility to the disaster area, a drone (UAV; Unmanned Arial Vehicle) system, which is equipped with a wireless detection module, was introduced. Using these framework, this study aims to provide the rescuers with effective rescue information by calculating 3-D location for buried persons based on the wireless and barometer sensor fusion.
\end{abstract}

\section{INTRODUCTION}

In the urban area, when the enormous disasters like huge collapse accidents of buildings and infrastructures such tunnel, subway etc. occurs, securing action technologies of initial disasters plays an important role in guaranteeing survival golden time for buried persons. Accordingly, quick and accurate location detection of the buried persons in collapsed site can be utilized as an essential technology for saving in any disaster environment.

In the past, in order to detect survival status of buried persons and rescue them, the rescuers such as fire fighter, police officers etc., are mainly utilizing rescue dogs and conventional detectors with direct contact type on collapsed surface. Since these detection methods are applied approaching directly by rescuers onto the surface, due to secondary collapse risks, additional life loss can be occurred. Besides, instructions of detection equipment are complicated, and there are many difficulties in utilizing such equipment. Of note, it takes long time to detect the buried persons. To solve these technical issues, we have chosen 'Drone', which is a kind of UAV (Unmanned Ariel Vehicle), in the spotlight that recently has widely been applied in most industrial market in order to detect and determine the position of the buried persons.

The aim of this study is to suggest a framework for detecting and identifying buried persons in collapsed disaster area with using a drone system embedded with Wi-Fi detection module we developed. Here, a drone system help us to perform a quick location positioning of the buried persons flying on the disaster area. The drone system should have advanced features that can capture and detect Wi-Fi signal emitted from burial sites.
The core technologies of the drone system that can detect buried persons includes Wi-Fi-based positioning techniques with GPS (Global Positioning System). Specially, in order to identify depth and plane position of the buried persons under pile of debris, the drone system should equip an $\mathrm{H} / \mathrm{W}$ module that can detect diverse signal strength like RSSI (Received Signal Strength Indication) from many sensors like Wi-Fi and barometer etc. embedded with mobile phones of buried persons. The drone system with the H/W module (Wi-Fi-based detection module) capture the signal and accurate location value with GPS according to the signal strength. Then, the drone system transfer such relevance data to the local computer through wireless communication. Therefore, in addition to the overall framework, this study suggest 2D positioning only with Wi-Fi (Kim et al. 2013) and 3D positioning with both Wi-Fi and barometer data. At this time, after analysing the signal and location data, we can visually identifying the $3 \mathrm{D}$ burial location of the buried persons according to the signal strength (Moon et al. 2015b).

This study has been kept researching since 2015 and will be finished next year. After that, it is expected that this technology will be utilized as a useful tool in order to rescue any buried and missing persons in disaster area.

\section{DRONE-BASED POSITIONING FRAMEWORK}

\subsection{Overall Framework}

In order to identify accurate $3 \mathrm{D}$ spatial position of buried persons, fusion techniques between diverse sensors such as Wi$\mathrm{Fi}$, GPS, barometer and others are required. It is difficult to determine accurate 3D location of buried persons with only Wi-

\footnotetext{
* Corresponding author
} 
Fi. Suppose that the buried persons have their mobile phone or the phones laid beside them. That is, exploring buried persons means identifying locations of $3 \mathrm{D}$ the mobile phone where it is. However, even if they have a mobile phone in disaster area, due to the stacked debris, a wireless signal strength emitted from their mobile phones can be inevitably weaken. Especially, WiFi signal has a characteristics that some are penetrated and some are emitted onto the ground through diffraction or reflection.

Since the Wi-Fi signal strength (RSSI) (Han et al. 2015) has a log pattern diminish by distance, it is identified that the location detection coverage is within about $60 \mathrm{~m}$ in an ideal opened space by a test result. However, since the collapsed site has rare space or closed, the signal might decrease sharply. Thus, in order to identify the captured signal, the location should be measured with only weak signal.

Especially, according to the signal feature of Wi-Fi, the 3D positioning by signal coverage is theoretically possible, but we can only identify 2D locations by detection coverage by signal strength. Therefore, in order to perform a 3D positioning process for buried persons, data fusion of diverse sensors should be made.

2D position in plane disaster area can be made with $\mathrm{Wi}$-Fi and GPS only. In addition to this, the 3D position for 3D spatial depth of buried person also can be determined with using barometer data from mobile phone.

\subsection{Constraints for Applying Drone-based Detection Techniques with a Wi-Fi Module}

There are several constraints in applying the drone-based survivor detection module to the real disaster area. At first, we suppose that any additional equipment excepting for a drone system equipped with GPS and Wi-Fi detection module should not be used. In addition to this, the buried persons should have the mobile phone with power on, and it should be placed beside them. That is, the mobile phones also should not be broken, and Wi-Fi is always activated. It is supposed that the mobile phones is also equipped with diverse sensors, which include Wi-Fi, Bluetooth and barometer etc., and 3D positioning by them should be possible with Wi-Fi only in environment where $3 \mathrm{G}$ and $4 \mathrm{G}$ communication is interrupted.

Of note, considering the wireless communication security that a smartphone has, the background application that enables Wi-Fi sensor activation remotely should be embedded. If there are not any supporting applications, the drone system have to scan the Wi-Fi signal and force them to activate. The mobile phone with an android platform is possible on this case, but them with iOS platform is not possible.

\section{2D POSITIONING FRAMEWORK WITH WIFI ONLY}

\subsection{Identification Test of Wi-Fi Detection Coverage}

This study developed an android platform-based sensor collector application that enables the mobile phone to collet sensor data regularly in order to identify detection coverage of $\mathrm{Wi}-\mathrm{Fi}$ signal. The collection target is based on $\mathrm{Wi}-\mathrm{Fi}$ and Bluetooth signal, and the application was configured to be gained as general collection mode. When the application is activated, a collecting process of sensor data is started.

In order to scan Wi-Fi signal data, the application executed scanning process creating Wi-Fi module, and collected log data can be represented in a form of time and sensor type on screen. The collection data of Wi-Fi sensor are stored into DB with start and end time of scanning process, and AP identification data. When completing the collection, data from DB are extracted for chosen sensors.

Using a sensor collector, we have performed a test-bed in order to analysed feasibility for diminution features of signal by distance identifying detection coverage of Wi-Fi signal in an ideal site. As a result, when identifying trend curve for each graph, it is recognized that the Wi-Fi signal is diminished in a $\log$ scale. Since signal transmission strength from mobile phones depends on the Wi-Fi module mounted in each phone, the difference ratio is little. This result can be utilized in a phase of feasibility test

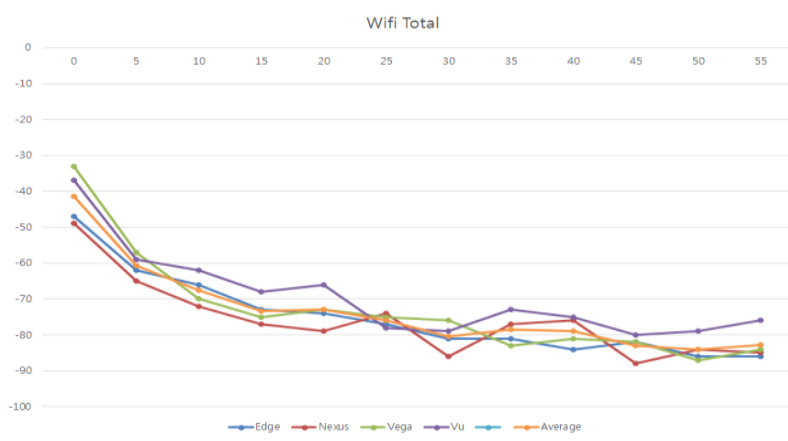

Figure 1. Wi-Fi signal curve by each smart phone

\subsection{D Positioning Model with Wi-Fi for Buried Persons}

In order to theoretically verify the signal strength change status for Wi-Fi by distance of each mobile phone, this study applied Wi-Fi technique for feasibility test of a $2 \mathrm{D}$ positioning algorithm for the mobile phones. At first, we have verified a hypothesis by collecting the real data on if $2 \mathrm{D}$ positioning is possible with Wi-Fi signal only in 2D plane or not. Figure 2 shows a process enables us to stochastically determine the location of the corresponding mobile phone by an access point (AP) on 2D plane.

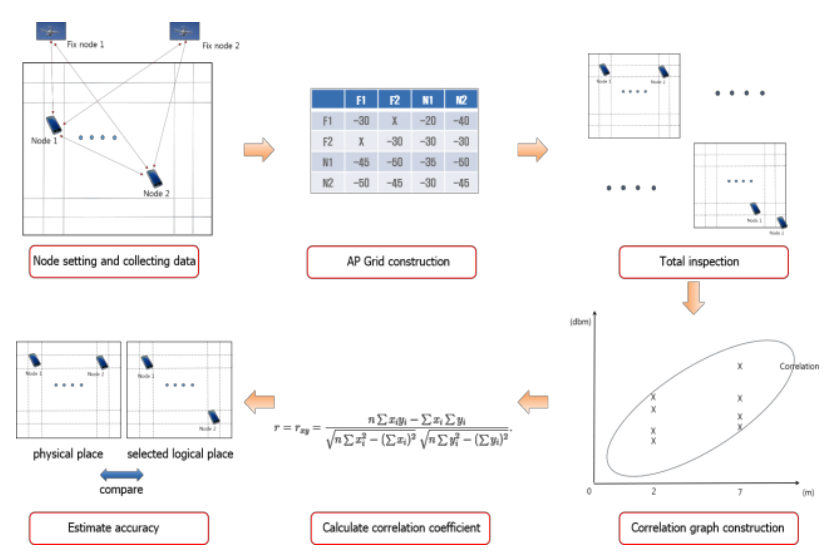

Figure 2. 2D positioning process by Wi-Fi

Firstly, a drone, which is a fixed node, and mobile phone of the buried persons is arranged into a virtual 2D space. Relationships on the signal and distance among nodes are analysed through collected data. Supposing that we already know the real location value, we review if measurement of logical locations is possible through total inspection or not. These data are used in calculating coefficient of correlation by each situation analysing a correlation analysis. At this time, the location accuracy is verified by comparing between initial node 
location and reals node location that express the most correlation value. At this time, LDPL (Log-distance path loss model) is applied.

\subsection{D Positioning Algorithm}

The test was performed in $10 \mathrm{~m} * 10 \mathrm{~m}$ unit on any playground area with open space, and each cell divided into $1 \mathrm{~m}^{*} 1 \mathrm{~m}$ unit. A sensor collector application was used in order to collect sensor data, and obtained in 3 minutes interval. One equipment, which is a fixed node, collects and store Wi-Fi signal data for another 4 equipment, which is mobile phone. Through this, the ground truth is determined, and AP grid for logical distance is performed through total inspection configuring relationship between distance and wireless signal to the ground truth as the AP grid. At this time, all the nodes for each cell move, and number of cases that represent the highest value are determined deriving correlation coefficients for each number of cases.

During this process, the total inspection of $1 \mathrm{M}$ is executed because 3 mobile phone exist. Out of these values, accuracy is approached comparing the node arrangement that has the highest value with correct node arrangement.

After this, correlation graph with distances ( $\mathrm{X}$ axis) that expresses signal difference ( $\mathrm{Y}$ axis) between 2 nodes is made. This can be visualized as change status of marked cells.

Figure 3 shows original arrangement (Left) of nodes and changed arrangement (Right) of nodes that has the highest value according to the total inspection.

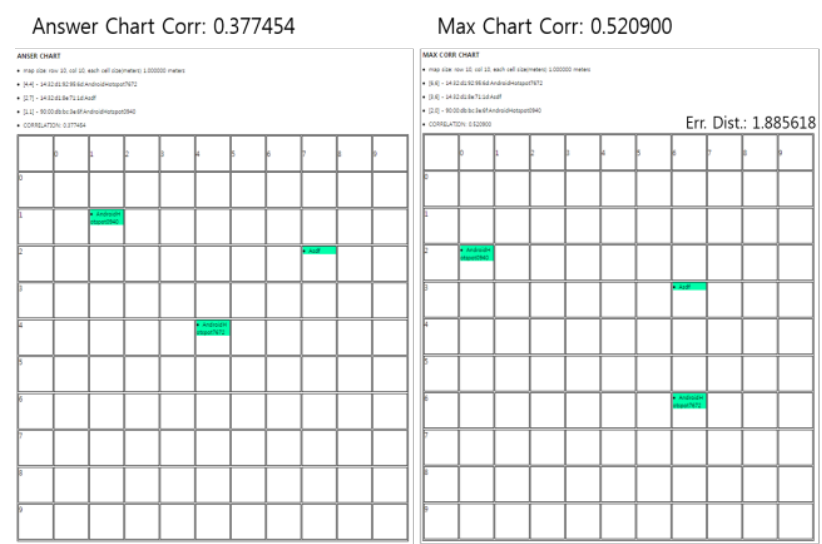

Figure 3. Node arrangement result after estimating accuracy test (Moon et al. 2015a)

Comparing with the 2 space grids, the distance difference between the changed and original space grid is $1.8 \mathrm{~m}$, and it is accurate considerably. It is judged that this expresses accuracy of a level of average 1.5 cell, which is very meaningful results. Since this case has 3 nodes which is the moving nodes, correlation calculation of $1 \mathrm{M}$ was performed. That is, the processing time to find the best solutions depends on the number of nodes, which represents mobile phones.

\section{3D POSITIONING BY SENSOR FUSIONS}

We have tested the $2 \mathrm{D}$ positioning algorithm in the previous section. However, it is difficult to identify burial depth with $\mathrm{Z}$ coordinates by $2 \mathrm{D}$ position of them only since the buried persons bury under the ground or covered debris. Thus, we suggest the methods on how we can estimate the depth with $\mathrm{Z}$ coordinate value using barometer sensor equipped with the mobile phones. Finally, through convergence of Wi-Fi and barometer sensors, the 3D burial coordinates of buried persons are determined.

\subsection{Height measurement by barometer sensor}

Relative positioning technologies like MEMS sensor-based PDR (Pedestrian Dead Reckoning) are more accurate position technologies against existing indoor positioning technologies. Since the relative positioning technologies are limited to the relative position recognition as the name represents itself, in order to this issue, existing absolute positioning technologies like Wi-Fi based positioning methods has been utilizing.

Barometer sensor has widely been applying as the techniques that relatively recognize movements between floors, but this study configures each floor data of the buildings as a database, and using this, suggests the technique that recognizes absolute floors consistently.

\subsection{D positioning algorithm by both $\mathrm{Wi}-\mathrm{Fi}$ and barometer}

The atmospheric pressure that changes over time can be revised through the pressure of the atmosphere at the sea level in given locations. Height results assume from 'P', which is the atmospheric pressure, collected from smart phones that keep the same heights should be constant ideally, but due to climate changes, the results are not uniform.

Errors of absolute atmosphere of MEMS sensor-based barometers, which equipped with smart phones, are $1 \mathrm{hPa}$, but errors of relative atmosphere is a level of $\pm 0.2 \mathrm{hPa}$. If this value is calculated into meter base, this means errors of about $\pm 2 \mathrm{~m}$ height. Here, in order to solve atmosphere differences that depend on the mobile phones, suggests methodologies that correct subtracting offsets, which are differences of atmosphere changes that depend on the time sequence, referring to the sealevel pressure and temperature from the Korea Meteorological Administration as the referenced data. In addition to this, in cases that an initial floor of the users was determined after storing height values by each floor, this study considers methodologies that enable us to detect accurate floor changes using the sea-level and temperatures in corresponding area.

Based on interaction formula of height-atmosphere, using ' $P$ ', which is the atmosphere measured by smart phones, ' $h$ ', which is floor height data, and ' $t m$ ', which is from the Korea Meteorological Administration, we can ' $P_{0}$ ' value that reflects locations of smartphones and errors with them. If there are height information of each floor, the atmosphere by floor can be predicted. The changing atmosphere that depends on the time sequences can be corrected through sea-level data in corresponding locations. The sea-level pressure is high in improvement effectiveness against corrections by temperatures. If the temperatures as the sea-level pressure are used, it is identified that the errors can be reduced into $4.5 \mathrm{~m}$ from $7 \mathrm{~m}$, which is the maximum delta.

\subsection{Feasibility test of 3D positioning algorithm}

Referring to the theory above, we have tested feasibility of the suggested theory in $3 \mathrm{D}$ space with $\mathrm{Z}$ axis for representing the depth for buried persons. When we look at the process on how we can estimate 3D spatial position of the buried mobile phones, firstly, the nodes are set, and Wi-Fi signal and barometer sensor data from the mobile phones are collected. In order to collect the atmosphere data from the nodes, which are mobile phone, an mobile application, which is the background app, we have developed, was utilized, and using this, the sensor data with Wi- 
Fi and barometer were gathered at intervals of $3 \mathrm{~m}$. Here, the concepts of fixed nodes, movement nodes, and space grid are the same with 2D positioning algorithm.

In the $2 \mathrm{D}$ test, total inspection of about $1 \mathrm{M}$ times was performed, but in this $3 \mathrm{D}$ test have a plenty of the total inspection. Thus, a problem of memory overflow occurred. In order to this issue, we have considered the algorithms so that 10 values with the highest correlation values only are stored. Through the constant updating, we have solved the memory overflow issue using a method that stores 10 items only with high rank.

When we identify correlation and error distances for each cases, in cases of utilization of Wi-Fi data and an ideal atmosphere data, the error distances are $5.67 \mathrm{~m}$. Whereas, if we use both Wi$\mathrm{Fi}$ and real barometer data, it is identified that the error distances are 5.88. It is recognized that using barometer data in estimating the locations is much more effective comparing to the Wi-Fi only.

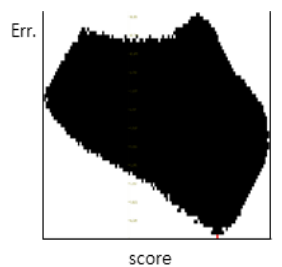

WiFi + ideal barometer

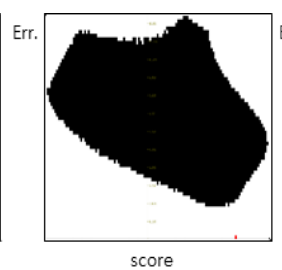

Wifi + real barometer

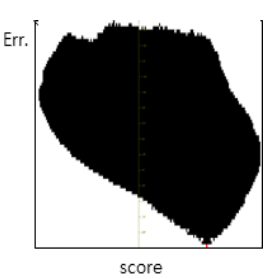

Wifi Only

\begin{tabular}{|l|r|r|}
\hline & \multicolumn{1}{|c|}{ Correlation } & Err. Dist. (m) \\
\hline WiFi + ideal barometer & 0.74 & 5.67 \\
\hline WiFi + real barometer & 0.64 & 5.88 \\
\hline WiFi Only & 0.84 & 7.06 \\
\hline
\end{tabular}

Figure 4. 3D positioning error distance with considering Wi-Fi and Ideal/Real barometer.

\section{CONCLUSIONS}

In this study, we have suggested the drone-based positioning framework with sensor fusion using Wi-Fi and barometer from the mobile phone in order to rescue the buried persons in case of the disaster. We have reviewed the $2 \mathrm{D}$ positioning algorithm with Wi-Fi only, and through sensor fusion with barometers, we have suggested the methodologies how we can estimate the 3D position considering the buried depth with $-\mathrm{Z}$ value.

Resulting the study, there are some limitations in terms of accuracy. Since the drone is using a general GPS, which is kind of A-GPS, or D-GPS, we cannot help depending on the GPS equipped with the drone. In order to this accuracy issues, we have to consider diverse algorithm, which includes kalman filter or other optimization algorithm that reduce the distance errors depend on the signal features. We will have a plan to use RTKGPS and the optimization algorithm this year, and improve the detection accuracy. Then, developing an $\mathrm{H} / \mathrm{W}$ module with $\mathrm{Wi}-$ Fi scanning (Rosati et al. 2013), we will equip them with the drone in order to detect the Wi-Fi signal (RSSI) flying on the disaster ground.

For future, it is expected that these technology will be utilized as core tools in order to save the human life in the era of drone.

\section{ACKNOWLEDGEMENTS}

This study was supported by "(16Main-Mission) Development of $3 \mathrm{D}$ model reconstruction technology of disaster area and location detection technology of survivors buried under the remains of the building" project funded by Korea Institute of Civil Engineering and Building Technology (KICT).

\section{REFERENCES}

Moon, H., Lee, W., Lee, G., and Han, D., 2015a. A 2-D Location Determination Model of Buried Persons in Collapsed Shape using Optimal Wireless Communication Technology, Journal of the Korea Academia-Industrial cooperation Society, 16(12), pp.8879-8888

Moon, H., Kim, C., and Lee, W., 2015b, "Wireless Communication-based Buried Person Detection Considering Characteristics of Collapsed Surface in Disaster Area", Proc. of 2015 Civil Expo \& Conference, Nov. 2015

Kim, S., and Han, D. 2013, "A study on constructing WiFi radio map by walking survey", Proc. of Korea Intelligent Transport System, pp. 155-158

Han, D., and Jeong, S., 2015, "Global Indoor Locating and Integrated Indoor/outdoor Navigation System", The Korean Institute of Communications and Information Sciences (Information and Communication), 32(2), pp. 89-97

S. Rosati, K. Kruzelecki, L. Traynard, and B. Rimoldi, 2013, "Speed-aware routing for uav ad-hoc networks," IEEE GLOBECOM 2013, 4th International IEEE Workshop on Wireless Networking \& Control for Unmanned Autonomous Vehicles: Architectures, Protocols and Applications, 2013 